\title{
Inertial bifurcation of the equilibrium position of a neutrally-buoyant circular cylinder in shear flow between parallel walls
}

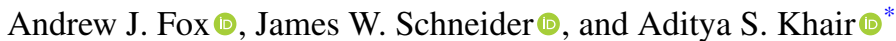 \\ Department of Chemical Engineering, Carnegie Mellon University, Pittsburgh, Pennsylvania 15213, USA
}

(Received 13 September 2019; published 3 January 2020)

\begin{abstract}
The motion of a neutrally buoyant, rigid circular cylinder in simple shear flow of a Newtonian fluid between parallel walls is calculated for various particle Reynolds numbers, $\operatorname{Re}_{p}=G a^{2} / v$, via the lattice Boltzmann method. Here, $G$ is the velocity gradient of the ambient shear, $a$ is the radius of the particle cross section, and $v$ is the kinematic viscosity of the fluid. An inertial lift force perpendicular to the ambient shear has a single zero crossing at the center of the channel below a critical $\operatorname{Re}_{p}$, corresponding to a single transverse equilibrium position. Above this critical $\mathrm{Re}_{p}$, the equilibrium position undergoes a pitchfork bifurcation, with an unstable zero-force equilibrium at the center and two equidistant stable equilibria off center. The trajectories of a force- and torque-free particle reach the stable equilibria independently of the initial particle position, with the exception of the aforementioned unstable equilibria. The critical $\operatorname{Re}_{p}$ increases with increasing confinement ratio $\kappa$ (i.e., the ratio of the radius of the particle cross section to channel width) and occurs below the transition to unsteady flow. Finally, we suggest how this inertial bifurcation could be used to develop novel particle separation techniques.
\end{abstract}

DOI: 10.1103/PhysRevResearch.2.013009

\section{INTRODUCTION}

Early observations of fluid inertia inducing particle migration across streamlines were reported by Segre and Silberberg $[1,2]$. In their study, poly(methyl methacrylate), or PMMA, spheres of density $\rho$ were suspended in mixtures of water, 1,3butanediol, and glycerol, the combination of which also had density $\rho$ (such that the particles were neutrally buoyant) and viscosity $\mu$. The suspension flowed through a circular tube of radius $R$ at a characteristic speed $U_{m}$. Their study showed that for flows of channel Reynolds number $\operatorname{Re}_{c}=\rho U_{m} R / \mu$ between 1 and 30 , particles migrated to an equilibrium position approximately $0.6 R$ from the axis, creating a "tubular pinch" effect. The phenomenon was caused by fluid inertia creating a force transverse to the flow, in what has become known as "inertial lift." This phenomenon only occurs at nonzero Reynolds numbers, as the linearity and reversibility of the Stokes equations prevents its occurrence in purely viscous flows. Interest in this phenomenon has increased recently with applications in microfluidic devices [3] due to the lack of external forces necessary to induce particle motion transverse to ambient flow. For example, inertial lift has been exploited to selectively separate blood cells [4], cancer cells [5], E. coli [6], and biodiesel-producing algae [7].

The experiments of Segre and Silberberg spurred many attempts to quantify inertial lift through asymptotic analysis at

\footnotetext{
*akhair@cmu.edu

Published by the American Physical Society under the terms of the Creative Commons Attribution 4.0 International license. Further distribution of this work must maintain attribution to the author(s) and the published article's title, journal citation, and DOI.
}

small (particle-scale) Reynolds numbers. Rubinow and Keller [8] investigated a sphere of radius $a$ translating at velocity $\boldsymbol{U}_{\boldsymbol{p}}$ and rotating at angular velocity $\boldsymbol{\Omega}_{p}$ in a quiescent fluid. This study used matched asymptotic expansions about small translational particle Reynolds numbers $\operatorname{Re}_{t}=\rho U_{p} a / \mu$, where $U_{p}=\left|\boldsymbol{U}_{\boldsymbol{p}}\right|$, to calculate a leading-order force transverse to the flow as $\boldsymbol{F}_{\boldsymbol{L}} \sim \pi a^{3} \rho \boldsymbol{\Omega}_{\mathbf{p}} \times \mathbf{U}_{\mathbf{p}}$. Saffman [9] investigated a sphere in unbounded simple shear (with velocity gradient $G$ ) translating along the streamlines of the undisturbed flow, with a "slip velocity" $\boldsymbol{U}_{\boldsymbol{s}}$ relative to the shear streamline passing through its center. He used matched asymptotic expansions to calculate that fluid inertia acts to deflect the particle toward streamlines opposite to the particle translation with a lift force of magnitude $F_{L} \sim 6.46 a \mu U_{s} \operatorname{Re}_{p}^{1 / 2}$ for small shear particle Reynolds number $\operatorname{Re}_{p}=\rho G a^{2} / \mu$. Cox and Brenner [10] considered particles of arbitrary shape (with characteristic linear dimension $a$ ) translating in planar Poiseuille flow at low particle Reynolds number a distance $d$ from the wall. A regular perturbation expansion of the Navier-Stokes equations about small particle Reynolds numbers and dimensionless particle size, or confinement ratio, $\kappa=a / d$ was used to develop an integral formula for the inertial lift force on a neutrally buoyant sphere and an equation for its transverse migration velocity. Ho and Leal [11] investigated neutrally buoyant spherical particles in bounded planar shear and Poiseuille flows. An analysis using the reciprocal theorem was conducted, expanding the disturbance velocity and pressure fields caused by the sphere in small $\operatorname{Re}_{p}$ and small confinement ratio $\kappa=a / R$. Calculations of the lift force acting on the particle revealed a zero crossing, corresponding to a transverse equilibrium position, at the center of the channel for shear flow and at 0.3 of the channel width from the center for Poiseuille flow. The latter is in agreement with Segre and Silberberg [1,2], although those experiments were in a tube Poiseuille flow. 
Schoenberg and Hinch [12] observed that while Ho and Leal [11] assumed $\operatorname{Re}_{c} \ll 1$, the channel Reynolds number in the experiments of Segre and Silberberg was not small; to reconcile these results, they investigated a sphere in Poiseuille flow at $\operatorname{Re}_{c}=O(1)$. They used a singular perturbation expansion in the limit of small $\kappa$ (and hence small $\mathrm{Re}_{p}$ ) to calculate the lift force on the sphere, via a finite-difference solution of the Fourier-transformed linearized Navier-Stokes equations, which govern the inertially dominated flow at the channel scale. Their results showed that the equilibrium position moved toward the wall of the channel as $\mathrm{Re}_{c}$ increased, in agreement with Segre and Silberberg [1,2].

Altena and Belfort [13] employed similar perturbation analyses to calculate the inertial lift on particles in channels with permeate flow through one wall, using expansions in terms of $\mathrm{Re}_{p}, \kappa$, and dimensionless permeate velocity relative to the pressure driven flow. Specifically, they calculated particle trajectories and equilibrium positions as functions of the dimensionless permeate velocity. Garcia et al. [14] also considered the inertial lift on particles in the presence of a permeate flow via numerical computations. Harding et al. [15] developed a model for inertial lift on spheres in curved ducts to predict the dependence of equilibrium positions of neutrally buoyant particles on channel bend radius.

More recent computational studies have performed numerical solutions of the Navier-Stokes equations to quantify the behavior of particles beyond the small $\mathrm{Re}_{p}$ regime. Feng et al. [16] studied a neutrally buoyant circular cylinder in planar shear flow between rigid walls. They used two-dimensional finite-element computations to calculate the particle trajectory at $\operatorname{Re}_{p}=0.625$ and $\kappa=0.125$. Their computations revealed that the particle migrated to an equilibrium position at the center of the channel. Pan et al. [17] also investigated a neutrally buoyant circular cylinder in confined shear flow over $1 \leqslant \operatorname{Re}_{p} \leqslant 10$. Their study used a fictitious domain method with distributed Lagrange multipliers to compute the flow around a cylinder. For $\operatorname{Re}_{p} \lesssim 2$ and $\kappa=0.125$, the particle migrated to an equilibrium position at the center of the channel. At $\operatorname{Re}_{p} \gtrsim 2$ and $\kappa=0.125$, the particle no longer migrated to the center of the channel, instead reaching an equilibrium position between the wall and centerline of the channel. This result showed that there exists a critical Reynolds number beyond which the particle can be directed to an off-center equilibrium position.

Here, we will investigate a neutrally buoyant cylinder in simple shear flow between parallel walls over the expanded regime $0.1 \leqslant \operatorname{Re}_{p} \leqslant 50$ and $0.0625 \leqslant \kappa \leqslant 0.25$. We will use the lattice Boltzmann method to calculate the force acting on the particle and its consequential inertial migration. First, by fixing the transverse position of the particle, the lift force acting on it will be calculated as a function of position at $\kappa=0.125$; this analysis will show that the inertial force acting upon a particle has multiple zero crossings above a critical $\mathrm{Re}_{p}$, resulting in an equilibrium position bifurcation. This bifurcation occurs while the flow is steady in the frame of the cylinder, i.e., below the Reynolds number at which transition to unsteady flow occurs [18]. Next, the trajectory of a forceand torque-free particle of $\kappa=0.125$ will be calculated, confirming the equilibrium position bifurcation. Finally, we will repeat this analysis for cylinders of $\kappa=0.0625$ and $\kappa=0.25$,

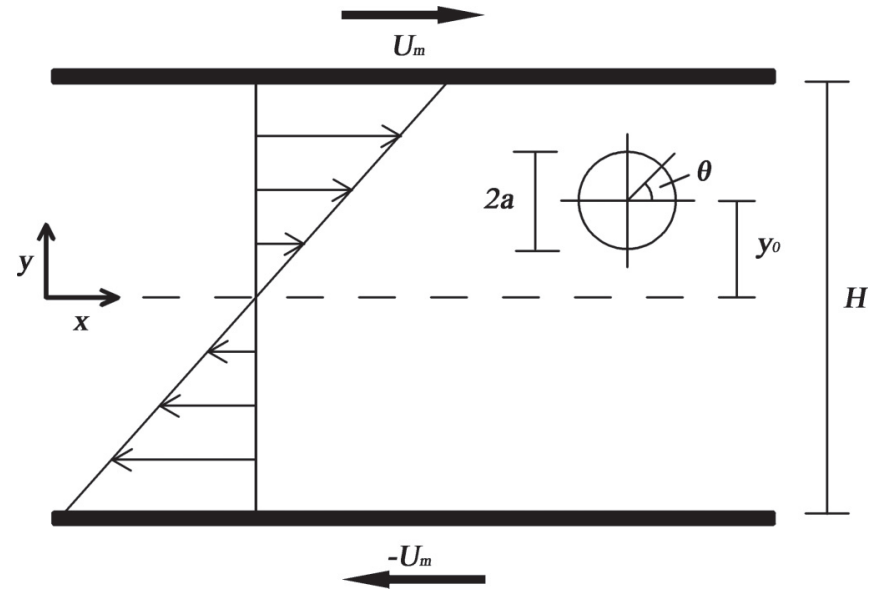

FIG. 1. A circular cylinder in two-dimensional shear flow between parallel walls. The behavior of the cylinder is determined by the initial transverse position $\tilde{y}_{0}=y_{0} / H$, the confinement ratio $\kappa=a / H$, and the particle Reynolds number $\operatorname{Re}_{p}=G a^{2} / \nu$, where $G=2 U_{m} / H$ is the velocity gradient. Here, $\theta$ is the angle of the position vector in the plane of the particle cross section relative to the imposed flow direction.

demonstrating that the critical $\operatorname{Re}_{p}$ decreases with decreasing $\kappa$. The remainder of this paper is organized as follows. In Sec. II, we outline the flow problem and calculations to be performed; in Sec. III, we describe the lattice Boltzmann method and verify the validity of our computational technique; in Sec. IV, we present results and discuss their implications; and in Sec. V, we deliver concluding remarks.

\section{PROBLEM FORMULATION}

Consider parallel walls at $y=-H / 2$ and $y=H / 2$, translating at speeds $U_{w}$ and $-U_{w}$, respectively, in the $x$ direction; this induces a shear flow in the Newtonian liquid between the walls with velocity gradient $G=2 U_{w} / H$. The fluid in the channel has a kinematic viscosity $v$. A neutrally buoyant circular cylinder of radius $a$ is placed within the channel at an initial dimensionless transverse position $\tilde{y}_{0}=y_{0} / H$. The dynamics of the cylinder are described by a particle shear Reynolds number $\operatorname{Re}_{p}=G a^{2} / \nu$, which characterizes the inertia of the shear on the scale of the particle, and confinement ratio $\kappa=a / H$. Figure 1 depicts the flow problem.

The effect of inertia on the particle motion is quantified through two types of calculation. First, the cylinder is fixed in the transverse $y$ direction, but allowed to freely rotate and translate in the flow $x$ direction. The force per unit length acting on the cylinder in the transverse direction is calculated to determine the dimensionless lift force $\tilde{F}_{L}=F_{L} / \rho U_{m}^{2} a \kappa^{2}$ at that position, where $\rho U_{m}^{2} a \kappa^{2}$ is the force per unit length scale. We repeated this process for several transverse positions $\tilde{y}_{0}$ to ascertain the lift force at a given $\operatorname{Re}_{p}$. Locations where $\tilde{F}_{L}$ vanishes correspond to transverse equilibrium positions; positions where the the force is positive below the zero-force location and negative above are stable equilibria, whereas positions with the converse are unstable equilibria. In the second type of calculation, the circular cylinder is force and torque free, allowing free translation and rotation. We 
calculated the trajectory of the particle to determine its transverse migration at a given $\operatorname{Re}_{p}$. The final position of the particle is a stable equilibrium, and was used to confirm observations of equilibrium bifurcation with increasing $\operatorname{Re}_{p}$ as found from the first type of calculation.

\section{LATTICE BOLTZMANN CALCULATIONS}

The inertial migration of the cylinder is quantified using the lattice Boltzmann (LB) method, which is an iterative technique for solving the Navier-Stokes equations that discretizes the fluid field into nodes [19]. At a given node, at position $\boldsymbol{x}$ and time $t$, there exists a distribution of particles $f_{\sigma i}(\boldsymbol{x}, t)$ with velocity $\boldsymbol{e}_{\sigma i}$ in the $\sigma i$ direction. The fluid particles move by the dimensionless LB equation [20]

$$
\begin{aligned}
f_{\sigma i}\left(\boldsymbol{x}+\boldsymbol{e}_{\sigma i}, t+1\right)= & f_{\sigma i}(\boldsymbol{x}, t)+\frac{1}{\tau}\left[f_{\sigma i}(\boldsymbol{x}, t)-f_{\sigma i}^{(\mathrm{eq})}(\boldsymbol{x}, t)\right] \\
& +\boldsymbol{g}_{\sigma i}(\boldsymbol{x}, t),
\end{aligned}
$$

describing the incremental time evolution of the fluid in the system. The LB method consists of alternating steps of particle collision and translation. Particle collision is represented by the second term on the right side of (1), wherein the current distribution of particles, $f_{\sigma i}(\boldsymbol{x}, t)$, is compared to an equilibrium distribution of the same macroscopic velocity, $f_{\sigma i}^{\text {(eq) }}(\boldsymbol{x}, t)$, over a relation time $\tau=(6 v+1) / 2$. Translation, where the particles at each node translate to the next-nearest nodes, is denoted by the left side of (1). The final term in (1) represents an additional forcing due to the fluid-solid interaction force [20], which will be discussed below. The LB equation has been shown to reduce to the Navier-Stokes equations at small Mach and Knudsen numbers [21-23], and has been shown to accurately model transient problems [24].

A LB code was developed in this study. The fluid field was discretized into a computational domain of Eulerian nodes $n_{x} \times n_{y}$, where $n_{i}$ is the number of nodes in the $i$ direction, with a channel aspect ratio of $\mathrm{AR}=n_{x} / n_{y}=4$. A standard bounce-back boundary condition was used to simulate the translating walls [19], and a periodic boundary condition was used on the open ends. The particle was simulated with a series of Lagrangian nodes arranged regularly over its boundary. The force $\boldsymbol{F}$ and torque $\boldsymbol{T}$ per unit length acting on the particle were calculated using an external boundary force (EBF) method [20], which calculates a fluid-solid interaction force acting on the fluid $\boldsymbol{g}_{\sigma i}(\mathbf{x}, t)$ and a corresponding force acting on the nodes at the surface of the particle. The particle velocity $\boldsymbol{U}_{\boldsymbol{p}}$ and angular velocity $\boldsymbol{\Omega}_{p}$ were calculated from Newton's equations of rigid-body motion [19],

$$
M \frac{d \boldsymbol{U}_{\boldsymbol{p}}(t)}{d t}=\boldsymbol{F}(t) \text { and } I \frac{d \boldsymbol{\Omega}_{p}(t)}{d t}=\boldsymbol{T}(t),
$$

where $M$ is the mass per unit length of the cylinder and $I$ is the moment of inertia per unit length. The computational method proceeds iteratively. The fluid begins in a quiescent state, and the simulation initiates with the walls being set into motion. The EBF method calculates the force acting between fluid and particle nodes, from which the total force and torque per unit length on the particle can be determined via a summation over the particle cross section. The particle translational and angular velocities are calculated, after which the particle is (a)

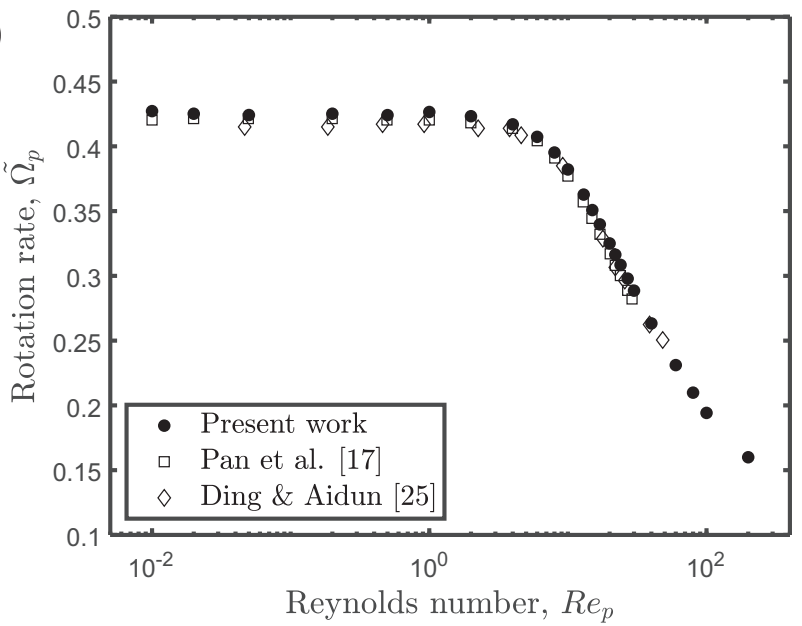

(b)

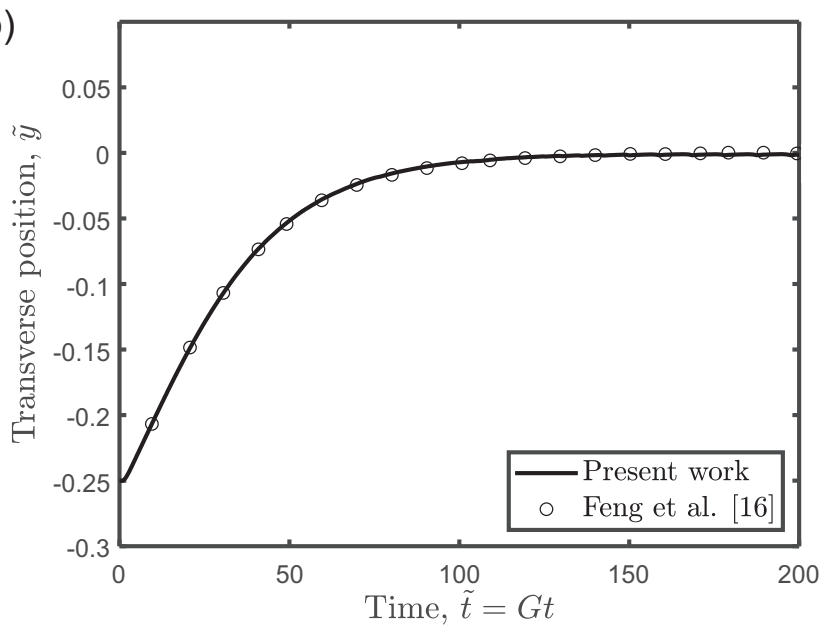

FIG. 2. (a) Rotation rate $\tilde{\Omega}_{p}$ of a circular cylinder with $\kappa=0.25$ at the center of the channel as a function of $\operatorname{Re}_{p}$. (b) Transverse trajectory of a circular cylinder with $\kappa=0.125$ and $\operatorname{Re}_{p}=0.625$ as a function of time. Relevant results from $[16,17,25]$ were digitized for replotting here.

translated and rotated. The fluid then undergoes collision and propagation, as previously discussed, and the iterative process repeats. This cycle continues until the force acting on the particle remains constant in the fixed $\tilde{y}_{0}$ studies or the particle reaches a constant $\tilde{y}$ in the free particle studies.

To validate the accuracy of our computational technique, a neutrally buoyant circular cylinder with $\kappa=0.25$ was simulated in shear flow at an initial position $\tilde{y}_{0}=0$, so that no lateral migration occurs. The particle is free to rotate, and the dimensionless rotation rate $\tilde{\Omega}_{p}=\Omega_{p} / G$, where $\Omega_{p}=\left|\boldsymbol{\Omega}_{p}\right|$, is calculated at varying $\operatorname{Re}_{p}$. The results are compared to the computations of Ding and Aidun [25] and Pan et al. [17]. As shown in Fig. 2(a), our simulations compare favorably with those previous studies. The final angular velocity of these calculations reached a fixed value at all $\operatorname{Re}_{p}$ and showed no time dependence, indicating that we are below the transition to unsteady flow. Our method was further validated by examining the migration of a circular cylinder with $\tilde{y}_{0}=-0.25$, $\kappa=0.125$, and $\operatorname{Re}_{p}=0.625$; these conditions match those studied by Feng et al. [16]. As shown in Fig. 2(b), our calculated trajectory matches their results. A channel aspect 


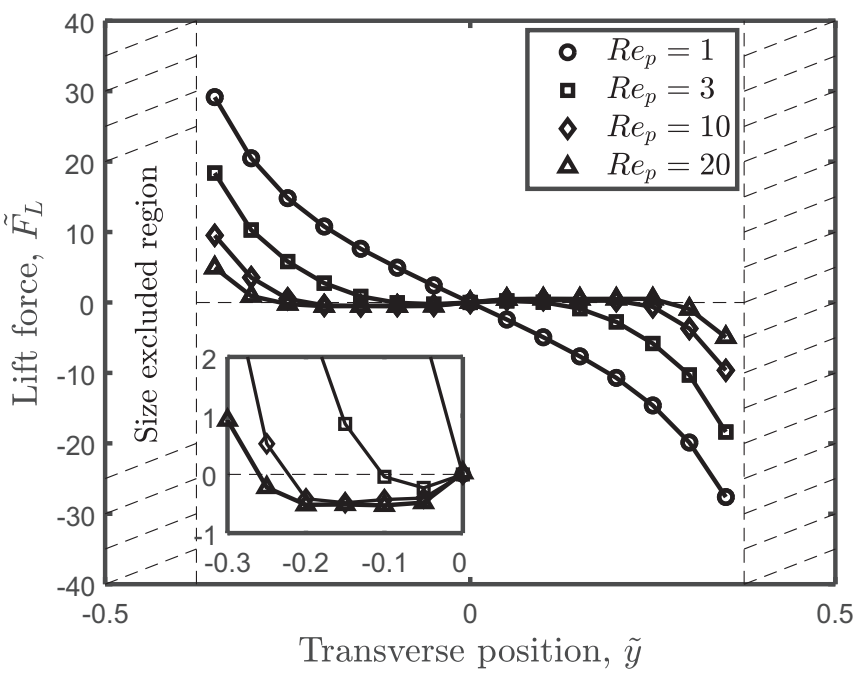

FIG. 3. Dimensionless lift force $\tilde{F}_{L}$ on a circular cylinder of $\kappa=0.125$ as a function of transverse position, with a magnification of the lift force just below the center of the channel in the inset. The finite particle radius precludes the center of the particle from entering the size excluded region.

ratio of $\mathrm{AR}=4$ was used in both verification studies; higher AR simulations were found to produce little change in the results.

\section{RESULTS AND DISCUSSION}

\section{A. Inertial lift force on a transversely fixed circular cylinder}

The inertial lift force per unit length on a circular cylinder of $\kappa=0.125$ in shear flow was calculated by fixing the transverse position $\tilde{y}_{0}$ of the particle. The particle was allowed to freely translate along the flow direction and rotate as the system evolved from its initial quiescent state. This process was repeated for various $\tilde{y}_{0}$ and $\mathrm{Re}_{p}$, and the results are shown in Fig. 3. Mikulencak and Morris [18] computed a transition to unsteady flow occurring at $\operatorname{Re}_{p}=85$ for a freely rotating circular cylinder of $\kappa=0.125$ at $\tilde{y}_{0}=0$; the flows in our simulations are at lower $\mathrm{Re}_{p}$, and only steady flow was observed in the frame of the translating cylinder. Inertial lift in unsteady flow, while undoubtedly interesting, is beyond the scope of this study. At $\operatorname{Re}_{p}=1$, the force decreases monotonically across the channel with increasing $\tilde{y}$, and the force has a single zero crossing at the center $\tilde{y}=0$. This zero crossing corresponds to a single stable equilibrium, due to the upward-driving force below $(\tilde{y}<0)$ and vice versa. At $\operatorname{Re}_{p}=3$, the lift force no longer varies monotonically with increasing $\tilde{y}$. In addition to the zero crossing at the center, two additional zero crossings occur at $\tilde{y} \approx-0.10$ and 0.10 . The zero crossing at the center is now an unstable equilibrium, due to the downward-driving force below $(-0.10 \lesssim \tilde{y}<0)$ and upward-driving force above $(0<\tilde{y} \lesssim 0.10)$; the new zero crossings correspond to stable equilibria. Thus, the system has undergone a pitchfork bifurcation between $\operatorname{Re}_{p}=1$ and $\operatorname{Re}_{p}=3$. By increasing the Reynolds number to $\operatorname{Re}_{p}=10$, the stable equilibria move closer to the walls at $\tilde{y} \approx-0.23$
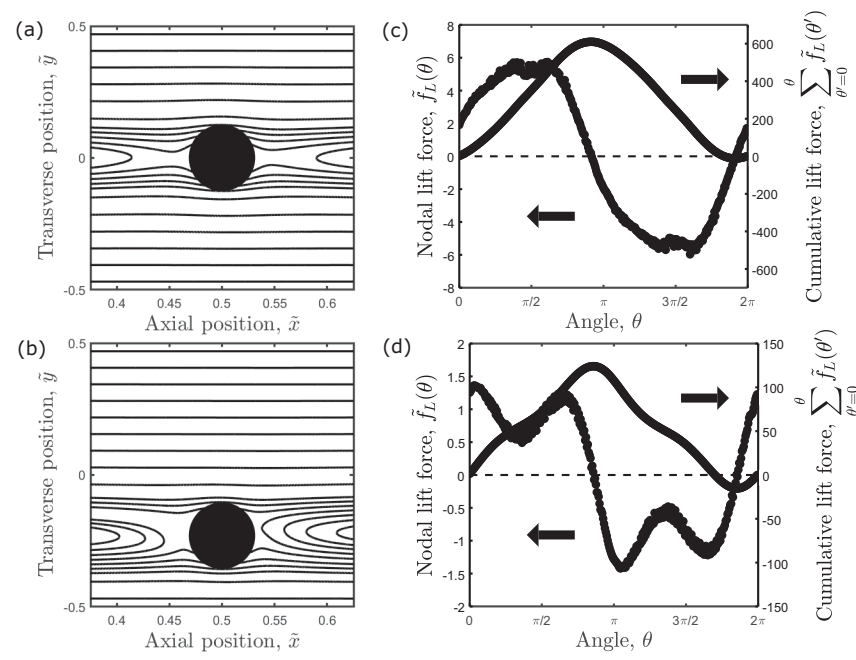

FIG. 4. Streamlines around circular cylinders of $\kappa=0.125$ at stable equilibrium positions in confined simple shear at (a) $\operatorname{Re}_{p}=1$ and (b) $\operatorname{Re}_{p}=10$. (c), (d) The lift force distribution and cumulative lift force about the cylinders.

and 0.23 ; the equilibrium positions shift further outward to $\tilde{y} \approx-0.27$ and 0.27 at $\operatorname{Re}_{p}=20$.

Unsurprisingly, from Fig. 3 the force $\tilde{F}_{L}$ at a fixed $\tilde{y}$ generally decreases in magnitude with increasing $\operatorname{Re}_{p}$, due to inertial screening of the velocity disturbance caused by the particle. Specifically, by "inertial screening" we mean that the velocity disturbance is confined to closer to the particle surface with increasing $\mathrm{Re}_{p}$, thereby reducing the particle-wall hydrodynamic interaction and lift force. For example, as $\operatorname{Re}_{p}$ increases from 1 to 20, the dimensionless lift force decreases from 29 to 4.9 close to a wall at $\tilde{y}=-0.35$. That screening reduces the wall-induced inertial lift force that drives the particle to the center of the channel; therefore, the $\tilde{F}_{L}$ vs $\tilde{y}$ curves appear flatter as $\operatorname{Re}_{p}$ increases. What is surprising, however, is that these curves have a zero crossing at offcenter transverse positions beyond a critical $\mathrm{Re}_{p}$, leading to the center switching from a stable to an unstable equilibrium position. Asmolov [26] demonstrated through matched asymptotic expansions that a neutrally buoyant sphere in linear shear flow bounded by a single wall would experience a lift force only directed away from the wall with no equilibrium position; the observed equilibrium position in our computations must therefore be caused by a second bounding wall.

To further understand the stable equilibria, the flow about the particle and nodal lift force distribution over its surface are shown in Fig. 4. Through the EBF method, the fluid-solid interaction force occurring at each node inscribing the particle surface is calculated. The force on each node in the $\tilde{y}$ direction is averaged over 1000 iterations to reduce discretizationinduced noise, producing a nodal lift force distribution as a function of surface angle $\tilde{f}_{L}(\theta)$ (Fig. 1); additionally, the cumulative lift force $\sum_{\theta^{\prime}=0}^{\theta} \tilde{f}_{L}\left(\theta^{\prime}\right)$ was plotted over the surface of the cylinder. While the distribution of the lift force changes, the sum of the $y$ component of the force acting on the surface of the particle at the equilibria, i.e., $\tilde{F}_{L}=\sum_{\theta^{\prime}=0}^{2 \pi} \tilde{f}_{L}\left(\theta^{\prime}\right)$, remains zero in both instances. 


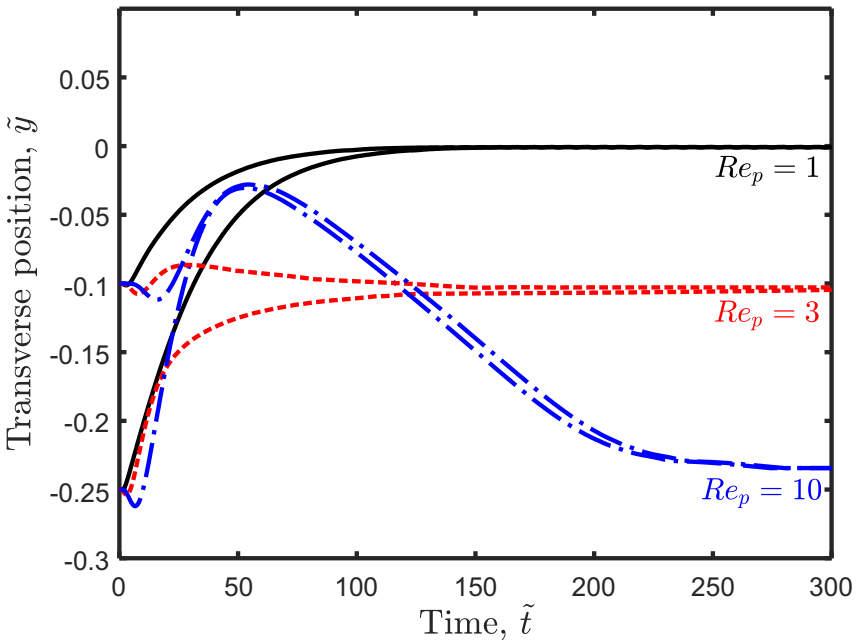

FIG. 5. Trajectories for a free circular cylinder of $\kappa=0.125$, showing change of transverse position as a function of time.

\section{B. Inertial migration of a free circular cylinder}

The migration of a circular cylinder of $\kappa=0.125$ was computed by allowing the particle to freely translate and rotate in the shear flow. The particle was placed at initial positions of $\tilde{y}_{0}=-0.25$ and -0.1 , and allowed to migrate to an equilibrium position; the exercise was repeated for several $\operatorname{Re}_{p}$. The results are shown in Fig. 5; the trajectory at $\operatorname{Re}_{p}=0.625$ was shown previously in Fig. 2. At $\operatorname{Re}_{p}=1$ the equilibrium position remains at the center of the channel, consistent with our calculations of the lift force showing a single stable equilibrium at $\tilde{y}=0$. At $\operatorname{Re}_{p}=3$ the equilibrium position again bifurcates, reaching a stable position at $\tilde{y} \approx-0.10$. The unstable equilibrium position is unobserved as the particle begins away from the center; simulations with the particle beginning at $\tilde{y}_{0}=0$ show the cylinder remaining at the center. This result is in agreement with our calculations of lift force in Sec. IV A, demonstrating the same stable and unstable equilibria. At $\operatorname{Re}_{p}=10$ the equilibrium position shifts to $\tilde{y} \approx-0.23$ again per Fig. 3 . The equilibrium position in all cases is independent of initial position, with the sole exception of the unstable equilibrium.

\section{Effect of confinement ratio on equilibrium position of circular cylinder}

To demonstrate the effect of the confinement ratio, the equilibrium position was calculated as a function of $\operatorname{Re}_{p}$ for three values of $\kappa$. The equilibrium position was computed by developing force-position plots at several $\operatorname{Re}_{p}$, per Sec. IV A, and was repeated for confinement ratios of $\kappa=0.0625,0.125$, and 0.25 ; the results are displayed in Fig. 6. The computations for $\kappa=0.125$ correspond to those discussed in Sec. IV A. At the smallest confinement ratio $\kappa=0.0625$, the equilibrium position bifurcation occurs when $\operatorname{Re}_{p} \approx 0.4$, lower than that of $\kappa=0.125$. While $\operatorname{Re}_{p}$ is small in this case, the channel Reynolds number at the bifurcation is not small: $\operatorname{Re}_{c}=\kappa^{-2} \operatorname{Re}_{p} \approx 102$. Increasing the confinement ratio to $\kappa=0.25$ requires a higher $\operatorname{Re}_{p}$ to induce the equilib-

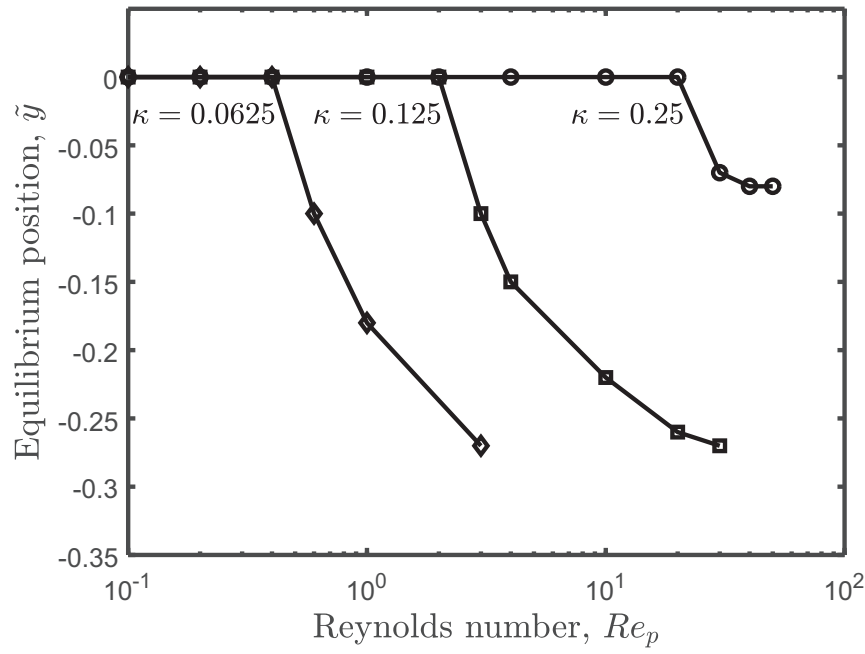

FIG. 6. Equilibrium position of a circular cylinder in confined shear flow as a function of $\operatorname{Re}_{p}$ for three confinement ratios.

rium position bifurcation. These results show that the particle equilibrium bifurcation is dependent on the confinement ratio.

\section{CONCLUSIONS}

In this study, the behavior of a neutrally buoyant circular cylinder in confined shear flow was quantified. The LB method was used to calculate the lift force and trajectories of a cylinder over $0.1 \leqslant \operatorname{Re}_{p} \leqslant 50$ and $0.0625 \leqslant \kappa \leqslant 0.25$. The stable equilibrium position of the cylinder undergoes a pitchfork bifurcation on increasing $\operatorname{Re}_{p}$ beyond a critical value dependent on $\kappa$. This is surprising given the geometric symmetry inherent to the problem. Specifically, below this value, the centerline is the sole equilibrium position; above, the centerline is an unstable equilibrium and two new offcenter equilibria emerge at equal distances above and below the center. The bifurcation occurs before the transition to unsteady flow, but is presumably the first in a cascade of events as $\operatorname{Re}_{p}$ is increased further. The critical $\operatorname{Re}_{p}$ required to induce the bifurcation increases with particle size, over the range of $\kappa$ examined.

Although in this study we considered an infinitely long cylinder, our preliminary computations on spherical particles suggest that the inertial bifurcation persists in three dimensions. This ongoing work will be reported in a future study. Importantly, such three-dimensional computations could be verified through laboratory experimentation using a parallel band apparatus [27,28]. Further studies would then consider the behavior of shape anisotropic particles, such as oblate and prolate spheroids. The asymmetry of these shapes introduce additional physics through orientation dynamics not seen for a cylinder or sphere. Spheroidal particles experience complex rotational dynamics, from Jeffery orbits at $\operatorname{Re}_{p}=0$ [29] to tumbling and rolling states at finite $\operatorname{Re}_{p}$ for prolate and oblate spheroids, respectively [30-32]. These additional physics would lead to more complex translational dynamics and would introduce additional factors impacting equilibrium position. 
Finally, equilibrium position bifurcations in shear flow present an opportunity for novel particle-particle separation techniques. Many current size-based inertial lift separation devices use pressure-driven flows to force particles to offcenter equilibrium positions, which can be weakly dependent on $\operatorname{Re}_{p}$ and $\kappa[14,33]$. For example, Kuntaegowdanahalli et al. [34] designed a device to segregate two neural stem cells, SH-SY5Y neuroblastoma $(a=8 \mu \mathrm{m})$ and C6 glioma cells $(a=4 \mu \mathrm{m})$; the total separation of these cells is necessary for properly identifying their individual functions and applications. Using a spiral microchannel, they were able to separate the cells at $80 \%$ efficiency. As the pitchfork bifurcation in shear flow occurs above a critical $\operatorname{Re}_{p}$ dependent on $\kappa$, performing a separation at a given $\operatorname{Re}_{p}$ would cause particles with critical $\operatorname{Re}_{p}$ below this value to segregate off center. By selecting the appropriate operating conditions, particles below a selected size would be separated from the larger ones, leading to a precise size-based separation. Using a parallel band device similar to that designed by Birkhofer et al. [35], a shear flow may be created that, assuming an equilibrium position bifurcation occurs, can be used to completely separate these cells. By operating with band speed of $0.9 \mathrm{~m} / \mathrm{s}$ and a gap width of $64 \mu \mathrm{m}$, the SH-SY5Y neuroblastoma cells $\left(\operatorname{Re}_{p}=\right.$ 0.45 and $\kappa=0.063$ ) would be moved off center by the shear flow, while the $\mathrm{C} 6$ glioma cells $\left(\operatorname{Re}_{p}=1.8\right.$ and $\left.\kappa=0.125\right)$ would remain at the centerline of the shear channel. Such a separation would offer high selectivity due to the strong dependence of equilibrium position on $\operatorname{Re}_{p}$, arising from the inertial bifurcation studied here.

\section{ACKNOWLEDGMENT}

This work was supported by a grant from the Pennsylvania Infrastructure Technology Alliance supplied by the Pennsylvania Department of Community and Economic Development.
[1] G. Segré and A. Silberberg, Radial particle displacements in Poiseuille flow of suspensions, Nature 189, 209 (1961).

[2] G. Segré and A. Silberberg, Behavior of macroscopic rigid spheres in Poiseuille flow, J. Fluid Mech. 14, 136 (1962).

[3] Y. Gou, Y. Jia, P. Wang, and C. Sun, Progress of inertial microfluidics in principle and application, Sensors 18, 1762 (1962).

[4] N. Nivedita and I. Papautsky, Continuous separation of blood cells in spiral microfluidic devices, Biomicrofluidics 7, 054101 (2013).

[5] S. C. Hur, A. J. Mach, and D. Di Carlo, High-throughput size-based rare cell enrichment using microscale vortices, Biomicrofluids 5, 022206 (2011).

[6] A. J. Mach and D. Di Carlo, Continuous scalable blood filtration device using inertial microfluidics, Biotechnol. Bioeng. 107, 302 (2010).

[7] M. Li, H. E. Munoz, K. Goda, and D. Di Carlo, Shape-based separation of microalga euglena gracilis using inertial microfluidics, Sci. Rep. 7, 10802 (2017).

[8] S. I. Rubinow and J. B. Keller, The transverse force on a spinning sphere moving in a viscous fluid, J. Fluid Mech. 11, 447 (1961)

[9] P. G. Saffman, The lift on a small sphere in a slow shear flow, J. Fluid Mech. 22, 385 (1965).

[10] R. G. Cox and H. Brenner, The lateral migration of solid particles in Poiseuille flow I. Theory, Chem. Eng. Sci. 23, 147 (1968).

[11] B. P. Ho and L. G. Leal, Inertial migration of rigid spheres in two-dimensional unidirectional flows, J. Fluid Mech. 65, 365 (1974).

[12] J. A. Schonberg and E. J. Hinch, Inertial migration of a sphere in poiseuille flow, J. Fluid Mech. 203, 517 (1989).

[13] F. W. Altena and G. Belfort, Lateral migration of spherical particles in porous flow channels: Application to membrane filtration, Chem. Eng. Sci. 39, 343 (1984).

[14] M. Garcia and S. Pennathur, Inertial particle dynamics in the presence of a secondary flow, Phys. Rev. Fluids 2, 042201(R) (2017).
[15] B. Harding, Y. M. Stokes, and A. L. Bertozzi, Effect of inertial lift on a spherical particle suspended in flow through a curved duct, J. Fluid Mech. 875, 1 (2019).

[16] J. Feng, H. H. Hu, and D. D. Joseph, Direct simulation of initial value problems for the motion of solid bodies in a Newtonian fluid. Part 2. Couette and Poiseuille flows, J. Fluid Mech. 277, 271 (1994).

[17] T. W. Pan, S. L. Huang, S. D. Chen, C. C. Chu, and C. C. Chang, A numerical study of the motion of a neutrally buoyant cylinder in two dimensional shear flow, Comput. Fluids 87, 57 (2013).

[18] D. R. Mikulencak and J. F. Morris, Stationary shear flow around fixed and free bodies at finite Reynolds number, J. Fluid Mech. 520, 215 (2004).

[19] C. K. Aidun, Y. Lu, and E. J. Ding, Direct analysis of particulate suspensions with inertia using the discrete Boltzmann equation, J. Fluid Mech. 373, 287 (1998).

[20] J. Wu and C. K. Aidun, Simulating 3D deformable particle suspensions using lattice Boltzmann method with discrete external boundary force, Int. J. Numer. Meth. Fluids 62, 765 (2010).

[21] G. R. McNamara and G. Zanetti, Use of the Boltzmann Equation to Simulate Lattice-Gas Automata, Phys. Rev. Lett. 61, 2332 (1988).

[22] H. Chen, S. Chen, and W. H. Matthaeus, Recovery of the Navier-Stokes equations using a lattice-gas Boltzmann method, Phys. Rev. A 45, R5339(R) (1992).

[23] S. Hou, Q. Zou, S. Chen, G. Doolen, and A. C. Cogley, Simulation of cavity flow by lattice Boltzmann method, J. Comput. Phys. 118, 329 (1995)

[24] T. Rosen, M. Do-Quang, C. K. Aidun, and F. Lundell, The dynamical states of a prolate spheroidal particle suspended in shear flow as a consequence of particle and fluid inertia, J. Fluid Mech. 771, 115 (2015).

[25] E. Ding and C. K. Aidun, The dynamics and scaling law for particles suspended in shear flow with inertia, J. Fluid Mech. 423, 317 (2000).

[26] E. S. Asmolov, The inertial lift on a spherical particle in a plane Poiseuille flow at large channel Reynolds number, J. Fluid Mech. 381, 63 (1999). 
[27] G. I. Taylor, The formation of emulsions in definable fields of flow, Proc. R. Soc. A 146, 501 (1934).

[28] A. C. Rust and M. Manga, Bubble shapes and orientations in low Re simple shear flow, J. Colloid Interface Sci. 249, 476 (2002).

[29] G. B. Jeffery, The motion of ellipsoidal particles immersed in a viscous fluid, Proc. R. Soc. A 102, 161 (1922).

[30] T. Rosen, F. Lundell, and C. K. Aidun, Effect of fluid inertia on the dynamics and scaling of neutrally buoyant particles in shear flow, J. Fluid Mech. 738, 563 (2014).

[31] T. Rosen, J. Einarsson, A. Nordmark, C. K. Aidun, F. Lundell, and B. Mehlig, Numerical analysis of the angular motion of a neutrally buoyant spheroid in shear flow at small Reynolds numbers, Phys. Rev. E 92, 063022 (2015).
[32] W. Mao and A. Alexeev, Motion of spheroid particles in shear flow with inertia, J. Fluid Mech. 749, 145 (2014).

[33] D. Di Carlo, J. F. Edd, K. J. Humphry, H. A. Stone, and M. Toner, Particle Segregation and Dynamics in Confined Flows, Phys. Rev. Lett. 102, 094503 (2009).

[34] S. S. Kuntaegowdanahalli, A. A. S. Bhagat, G. Kumar, and I. Papautsky, Inertial microfluidics for continuous particle separation in spiral microchannels, Lab Chip 9, 2973 (2009).

[35] B. H. Birkhofer, J.-C. Eischen, D. Megias-Alguacil, P. Fischer, and E. J. Windhab, Computer-controlled flow cell for the study of particle and drop dynamics in shear flow fields, Ind. Eng. Chem. Res. 44, 6999 (2005). 